Itinéraires Itinéraires

Littérature, textes, cultures

2010-2 | 2010

Les blogs

\title{
Reconfigurations de la critique littéraire dans les blogs d'écrivains
}

\section{Brigitte Chapelain}

\section{(2) OpenEdition}

Journals

Édition électronique

URL : http://journals.openedition.org/itineraires/2040

DOI : $10.4000 /$ itineraires. 2040

ISSN : 2427-920X

Éditeur

Pléiade

\section{Édition imprimée}

Date de publication : 1 juillet 2010

Pagination : 131-140

ISBN : 978-2-296-12012-9

ISSN : $2100-1340$

\section{Référence électronique}

Brigitte Chapelain, "Reconfigurations de la critique littéraire dans les blogs d'écrivains », Itinéraires [En ligne], 2010-2 | 2010, mis en ligne le 01 juillet 2010, consulté le 21 avril 2019. URL : http://

journals.openedition.org/itineraires/2040 ; DOI : 10.4000/itineraires.2040

\section{(ब) $(\Theta \Theta$}

Itinéraires est mis à disposition selon les termes de la licence Creative Commons Attribution - Pas d'Utilisation Commerciale - Pas de Modification 4.0 International. 


\title{
Reconfigurations de la critique littéraire dans les blogs d'écrivains
}

\begin{abstract}
The hypothesis of a reappraisal of literary criticism on writers' blogs is examined from a literary and communicational stand point. It is born especially from a scaling down of traditional categories of literary criticism by an addition of generic structures linked with several languages used, by a «meta-mediation » of literary learnings as well as a « re-documentarizing process » offered to the Internet user.
\end{abstract}

Keywords : literary criticism, writer's blogs, media coverage, intertextuality, metamediation, re-documentarizing process

Mots clés : critique littéraire, blog d'écrivains, médiatisation, intertextualité, métamédiation, re-documentarisation

Francis Pisani souligne dans son blog, en mai 2007, une tendance dans la presse américaine à supprimer le critique littéraire et les pages consacrées à la littérature devant l'importance du phénomène suscité par les blogs ${ }^{1}$. Le 20 mai 2007, Pierre Assouline ${ }^{2}$ évoque une tribune de Richard Schickel dans le Los Angeles Times, dont le titre, «Tout le monde n'est pas un critique », rappelle que le critique littéraire est un professionnel. De nombreux commentaires réagissent à la nouvelle problématique que pose la médiatisation de la critique littéraire sur les blogs. Comment s'y est-elle médiatisée? En quoi le genre et la pratique en sont-ils transformés?

Ce phénomène n'est guère interrogé, ni du côté des sciences de la littérature, ni du côté des sciences de l'information et de la communication.

1. Francis Pisani, « Fin des critiques littéraires », Transnets, 3 mai 2007, http://pisani.blog. lemonde.fr/2007/05/03/fin-des-critiques-litteraires/, consulté le 13 avril 2010.

2. Pierre Assouline, « Contre la critique citoyenne », La république des livres, 20 mai 2007, http://passouline.blog.lemonde.fr/2007/05/20/contre-la-critique-citoyenne-suite/, consulté le 13 avril 2010. 
C'est dans le cadre de cette interdisciplinarité que nous avons choisi de le faire. Notre réflexion s'appuiera sur l'observation et l'analyse d'une catégorie bien particulière de blogs littéraires : les blogs d'écrivains.

L'hypothèse privilégiée est celle d'une reconfiguration de la critique littéraire, c'est-à-dire celle d'une réorganisation communicationnelle et d'une reconstruction générique et informationnelle.

\section{Une nouvelle inscription communicationnelle}

François Bon nous donne une des meilleures définitions d'un blog d'écrivain qui « doit faire passer l'auteur qui s'y livre de l'expression de soi à l'expérience bien plus âpre du travail d'écrire ${ }^{3} »$.

Une vingtaine de ces blogs d'écrivains ${ }^{4}$ a été retenue pour les différents degrés d'interactivité offerts au lecteur, et non pour la quantité des commentaires déposés. Entre le blog de Michel de Castillo ne permettant pas de laisser de commentaires, et ceux de Pierre Assouline et d'Alain Mabanckou avec des commentateurs nombreux et fidèles, dont certains écrivent également dans d'autres blogs, différentes catégories se présentent.

Nous allons examiner sur ces blogs les éléments nouveaux qui caractérisent la critique littéraire du point de vue de l'énonciation, des textes et des processus génériques.

\section{Les différents lecteurs de ces blogs}

Le lectorat de ces blogs est composé de plusieurs catégories :

- les lecteurs destinataires supposés : ce sont les lecteurs habitués qui laissent régulièrement des commentaires, mais aussi les écrivains blogueurs et des lecteurs fidèles intéressés par la littérature qui peuvent devenir des commentateurs occasionnels ou habitués ;

- les lecteurs potentiels : un public curieux de la médiatisation du littéraire sur Internet, amateur de littérature, mais aussi les chercheurs et les internautes; - les lecteurs destinataires effectifs : il s'agit des commentateurs. On peut penser que cette catégorie lit à la fois l'ensemble des billets de l'écrivain et une bonne partie des commentaires des autres. Ils sont les auteurs de leurs textes et participent à la dynamique du blog.

Pierre Assouline a rassemblé certains commentaires dans un ouvrage intitulé Brèves de blog 5 . L'utilisation du pseudonyme, sans aucune place laissée au corps et à la graphie, donne une place privilégiée à l'écriture qui est essentiellement l'outil de communication. Le lecteur-commentateur

3. François Bon, « Internet et fantasmatique de l'écriture », Technikart, hors-série « Littérature », numéro spécial, mars 2007.

4. La liste de ces blogs se trouve à la fin de l'article.

5. Pierre Assouline, Brèves de blog, Paris, Les Arènes, 2008. 
devient critique et développe dans l'échange des analyses et des réflexions sur la littérature, attestant ainsi l'existence de nouvelles énonciations de la critique littéraire.

Des réactions primesautières, mais aussi des mises en commun et des partages d'informations nourrissent les interventions des lecteurs commentateurs. Les thèmes qui ont suscité les réactions les plus nombreuses concernent la traduction, l'histoire, et la fiction avec Les Bienveillantes et l'œuvre d'Alain Robbe-Grillet.

Dans le blog d'Alain Mabanckou, les Africains francophones et anglophones constituent une communauté de commentateurs très présente dans des débats comme celui de la littérature monde, ou des événements historiques, comme la mort du poète Aimé Césaire.

\section{L'intertextualité dans les billets des écrivains}

L'intertextualité des billets des écrivains ne désigne pas seulement la reprise du texte des autres dans sa propre écriture : elle relève du dialogisme ${ }^{6}$ qui, plus qu'un échange entre deux personnes, se pose comme la transformation d'une question en une réponse, et vice versa, ou encore la traduction de l'énonciation de l'autre pour mieux lui répondre?

Ces procédés d'intertextualité se retrouvent dans des interpellations d'écrivains à écrivains, avec reprises de questions posées et tentatives, ou apports, de réponses (Michel del Castillo à Pierre Assouline, Alain Mabanckou à Pierre Assouline, le Stalker à Jean-Luc Bitton et Marc Alpozzo). Ces phénomènes d'intertextualité et de dialogisme sont une preuve de la médiatisation spécifique des blogs qui multiplie les instances d'énonciation. Beaucoup d'écrivains ouvrent leur espace à d'autres écrivains, à des personnalités de la culture, à la reproduction d'articles de presse, à des larges citations reprises dans l'actualité, et à des commentateurs. Alain Mabanckou, Stéphane Berthomet, Jean-Luc Bitton, Raymond Alcovère en sont des exemples.

Dans beaucoup de blogs, les écrivains s'effacent en ne traitant, ou en n'analysant, que des œuvres ou des propos d'autres écrivains. Gérard Genette désigne par «métatextualité » cette forme de textualité qui est de commenter le texte d'un autre. Jean-Luc Bitton qui consacre son blog à Jacques Rigaut dont il écrit la biographie en est une illustration.

D'autres blogs offrent à la lecture de véritables appareils critiques et entretiennent avec les œuvres des relations paratextuelles, comme en témoignent certains billets de Pierre Assouline, Juan Asensio, Jean-Luc

6. Mikhaïl Bakhtine, Esthétique et théorie du roman, Paris, Gallimard, 1978.

7. Lucia Santaella-Braga, «Penser l'interactivité à la lumière du dialogisme », dans Serge Proulx, Louise Poissant et Michel Senecal (dir.), Communautés virtuelles. Penser et agir en réseau, Laval, Presses de l’Université Laval, 2006, p. 147-164. 
Bitton, François Bon. L'architextualité est ce type de relations qui s'établissent entre des billets singuliers et les statuts génériques dont ils relèvent. On évoquera le blog de Pierre Cousin fait de courts poèmes qui font penser à des haïkus, et celui de Jean-Luc Aotret qui remet à la mode des genres de poésie médiévale comme la baguenaude.

\section{De l'intertextualité à l'hypertextualité dans les blogs}

Pour François Bon, le blog permet une évolution vers ce qui lui semble essentiel au travail et à la réflexion sur la littérature : un lieu d'écriture en constant renouvellement avec carnets de liens, archives et informations. Le système de syndication permet avec un format RSS de suivre un grand nombre de sites et de blogs en étant informé des nouveautés. Les trackbacks permettent de ranger les billets du même blog, ou de blogs différents parlant du même sujet. Ajoutons à ces outils quelques moteurs de recherche internes sur certains blogs ${ }^{8}$.

Les blogs d'écrivains sont devenus multimédias : ils ont intégré la photo, le vidéoblog et l'audioblog. La majorité d'entre eux utilise toutes ces variétés d'outils et de langages. Les liens intégrés par les écrivains dans leurs billets, et les liens sélectionnés avec d'autres sites d'écrivains, sites et blogs littéraires sont les principaux procédés de l'écriture hypertextuelle. Cette hypertextualité permet à la fois de mieux s'informer, de se documenter, de visualiser des événements et des visages, de découvrir des revues et des ouvrages lors de leur parution et d'accéder à des savoirs nouveaux.

\section{Processus génériques de la critique littéraire}

S'interroger sur le genre du blog d'écrivains, c'est s'interroger aussi sur celui des billets et des commentaires, et quatre dimensions doivent être prises en compte : la dimension communicationnelle, la dimension langagière, la dimension discursive et la dimension littéraire et journalistique.

La démarche technique et la scène d'énonciation propres au blog sont celles de la correspondance.

Nous avons vu précédemment que le langage écrit se trouve souvent complété ou remplacé par le son, l'image et l'hypertextualité, qui rompent avec les traditions monolangagières et «unimédia» de la critique traditionnelle.

Dominique Maingueneau, en le qualifiant de constituant, considère le discours littéraire comme un archeion $^{9}$, c'est-à-dire à la fois source, autorité et archive. Ces discours constituants que sont les billets des écrivains

8. Par exemple ceux d'Irène Delse, Pierre Assouline, Dominique Autié.

9. Dominique Maingueneau, Le Discours littéraire. Paratopie et scène d'énonciation, Paris, Armand Colin, 2004. 
relèvent d'une organisation textuelle formant une totalité discursive. Les genres de discours sont immuables, mais ils contrastent avec des catégories littéraires qui les constituent. Les billets offrent des registres de discours variés qui intègrent des textes génériquement distincts. Par exemple, certains d'entre eux se rangent dans le registre didactique avec des textes ou des fragments de textes qui ne sont autres que des manifestes, des biographies ou des essais; le registre polémique regroupe des fragments ou des entités textuelles qui sont de la satire, de la controverse ou du pamphlet. Des catégories plus journalistiques viennent se greffer et rajouter à la complexité générique des textes. Entretiens, reportages et chroniques en constituent des éléments.

\section{Enjeux informationnels et culturels des blogs d'écrivains}

Les blogs d'écrivains mettent à disposition de l'internaute des contenus informationnels et cognitifs qui constituent le deuxième aspect de la reconfiguration de la critique littéraire sur Internet.

\section{L'information et l'analyse littéraires}

La présentation et l'analyse critique d'ouvrages récents ou anciens, écrits en français ou traduits, sont présentes dans tous les billets observés. Celles-ci s'écrivent différemment : Roland Fuentes ${ }^{10}$ offre des présentations descriptives classiques avec parfois une image de quatrième de couverture; Pierre Assouline, à l'occasion de la parution des œuvres de Georges Simenon dans la collection « Bibliothèque de la Pléiade ${ }^{11}$ », présente et analyse le roman Pedigree (1948) comme la matrice et l'éclairage révélés à mi-parcours de cette œuvre; François Bon reprend la présentation décalée de Mathias Enard et de son livre Zone ${ }^{12}$ publié sur le blog de l'écrivain et traducteur Caro ${ }^{13}$; Marc Villemain reprend un article qu'il a fait paraître dans le Magazine des livres et nous explique les raisons pour lesquelles il considère que le dernier ouvrage de Juan Manuel de Prada ${ }^{14}$ est un chef-d'œuvre. Alain Mabanckou présente le futur ouvrage L'Énigme $d u$ retour de son ami Dany Laferrière dans un contexte plus personnel et développe une réflexion sur le processus de son écriture alors qu'il commence lui-même un roman; et enfin Loïc Barrière présente un diaporama des couvertures des derniers livres qu'il a aimés, suscitant ainsi chez le lecteur une découverte d'abord visuelle des livres.

10. Son blog est intitulé Brouillon littéraire et artistique.

11. Sous la direction de Jacques Dubois et Benoît Denis.

12. Le livre a obtenu le prix Inter 2009.

13. Son blog est intitulé Le Clavier cannibale.

14. Le Septième Voile, Paris, Seuil, 2009. 
L'actualité littéraire est prise en compte sur plusieurs plans : éditorial comme la vente d'un exemplaire de l'édition originale d'Ulysse, ou l'embargo opéré par la veuve de Jorge Luis Borges sur une réédition de l'œuvre du grand écrivain dans la Pléiade; historique, avec l'administration d'un prix, ou la disparition d'écrivains qui donnent lieu à des portraits, des retours sur l'œuvre et l'écriture : ce fut le cas pour le Nobel de Le Clézio et la mort d'Aimé Césaire dont l'engagement et l'œuvre furent unanimement évoqués et longuement discutés; technique, comme l'annonce de l'EBM (l'expresso book machine), proposant près d'un million de titres à la demande, et les avancées du livre numérique; sociologique, comme la liste des livres les plus lus, ou la remise en question des prix littéraires; scientifique avec l'évocation et la discussion autour de certains colloques, et théories littéraires; mais aussi plus rarement anecdotique et « germanopratine », comme la querelle entre Marie Darrieussecq et Camille Laurens ou la parution en livre de la correspondance électronique de Michel Houellebecq et Bernard-Henri Lévy.

L'actualité littéraire permet de développer des problématiques qui nourrissent la réflexion et l'analyse : la notion de plagiat, l'exploitation du fait divers en littérature, la littérature et Internet, la littérature et la langue, le roman, et tant d'autres.

Le travail de l'écriture est également un thème récurrent dans les billets de ces écrivains qui s'expriment soit à travers l'analyse d'autres écrivains soit, de façon plus personnelle, par la référence à leur propre création.

\section{La réactualisation du débat et de la controverse}

Le débat littéraire désigne les discussions suivies et contradictoires sur une question, ou une problématique entre commentateurs et écrivains, mais aussi entre écrivains : des débats sur des grands sujets comme la traduction, l'écriture et son rapport avec la langue d'origine, les rapports de la littérature et du politique.

Des controverses ${ }^{15}$ ont été soutenues : leur intérêt est qu'elles ne sont jamais closes et qu'elles génèrent à chacune de leurs résurgences de nouveaux apports d'arguments et d'analyse. Une configuration triadique constituée par deux parties et un public pris à témoin anime la controverse qui est provoquée soit par un choc disruptif dû par exemple à une provocation au niveau du contenu, soit à un bouleversement des cadres de discussion ou à une « intensification des procédures dialogiques ordinaires ${ }^{16} »$.

15. En particulier celle initiée par la parution des Bienveillantes en 2006 sur l'histoire et la fiction, et celle concernant la « littérature-monde » en mars 2007.

16. Jean-Louis Fabiani, « Disputes, polémiques et controverses dans les mondes intellectuels. Vers une sociologie historique des formes de débat agonistique », Mil neuf cent, $\mathrm{n}^{\circ} 25$ : 
Les acteurs de la controverse mobilisent des ressources auxquelles on ne fait pas toujours appel dans des débats traditionnels. Il s'agit de ressources humaines (acteurs, écrivains, chercheurs) et non humaines (documentation, théories) dont on se réclame, ou que l'on va remettre en question et reconstruire. Le manifeste " Pour une "littérature-monde" en français ${ }^{17}$ » annonce la mort de la littérature francophone et promulgue la naissance d'une littérature-monde en français, où la langue «libérée de son pacte exclusif avec la nation » ne reconnaîtrait que le pouvoir de la poésie et de l'imaginaire. Fait nouveau, le débat s'est inscrit sur Internet, et plus particulièrement dans les blogs d'écrivains de Pierre Assouline et d'Alain Mabanckou ${ }^{18}$. Les ressources mobilisées montrent une grande connaissance chez les commentateurs des écrivains francophones. Au fil de la controverse, le doute se généralise sur le concept de «monde » ajouté à la littérature pour montrer son indépendance vis-à-vis de la France, et les principales questions se portent au final sur l'écriture et la langue française.

\section{Nouvelles fonctions de la critique littéraire}

Les blogs observés rassemblent des déclinaisons éditoriales variées de la critique littéraire. Les écrivains reprennent à leur compte et intègrent la critique littéraire provenant d'autres médias comme la presse écrite, la radio, l'émission de télévision et le documentaire. Celle-ci s'écrit ainsi dans un nouveau langage constitué par les spécificités du multimédia et de l'hypertextualité. On peut parler de méta-médiation de la critique littéraire.

Les lecteurs réguliers ont ainsi accès à une redocumentarisation du littéraire sur Internet. « Redocumentariser ${ }^{19}$ », c'est laisser à un bénéficiaire la possibilité de réactualiser des contenus sémiotiques selon ses usages. Les écrivains mettent ainsi en accès des archives, réutilisent des séquences audiovisuelles et des photos. Jean-Luc Bitton réunit au fil des jours dans son blog, qu'il désigne comme un «work in progress », toute la documentation multimédia utile qu'il analyse et commente pour écrire la biographie de Jacques Rigaut à laquelle il se consacre depuis quatre ans.

L'histoire littéraire renouvelle ces dernières années son approche en essayant de se rapprocher de la poétique ${ }^{20}$. Aux disciplines traditionnelles

« Comment on se dispute. Les formes de la controverse », mai 2007, p. 45-60.

17. Manifeste signé par quarante-quatre écrivains et publié dans Le Monde le 16 mars 2007. Michel Le Bris et Jean Rouaud (dir.), Pour une littérature monde, Paris, Gallimard, 2007.

18. Brigitte Chapelain, « Les blogs d'écrivains : un nouvel enjeu pour les littératures francophones », dans Larbi Chouikha, Vincent Meyer et Wahid Gdoura (dir.), Interagir et transmettre, informer et communiquer : quelles valeurs, quelles valorisations?, actes du colloque international des sciences de l'information et de la communication, Tunis, ISD, IPSI, SFSIC, 2008.

19. Jean-Michel Salaün, « La redocumentarisation, un défi pour les sciences de l'information », Études de communication, ${ }^{\circ}$ 30, 2007, p. 13-23.

20. Luc Fraisse (dir.), L'Histoire littéraire à l'aube du vingtième siècle : controverses et 
comme la bibliographie, l'histoire du livre et de sa représentation, l'histoire de la circulation des savoirs littéraires, de nouvelles perspectives rajoutent d'autres champs scientifiques comme l'écrivain et les penseurs contemporains, l'évolution des genres littéraires et l'histoire littéraire du lecteur. De même une réflexion sur les outils a été amorcée comme les entretiens d'auteur et les textes épistolaires qui transformeraient la relation auteur/lecteur et devraient être analysés d'un autre point de vue que celui de la prévarication ${ }^{21}$. L'histoire littéraire telle qu'elle évolue intègre des formes plus communicationnelles de la littérature. La critique littéraire exercée dans ces blogs fournit à l'histoire littéraire des données documentaires variées dans l'échange et le débat. Les blogs que nous avons analysés sont des données documentaires qui sont à prendre en compte dans ce renouveau de l'histoire littéraire soit par les contenus offerts, soit par la variété langagière et générique des méthodes de recueil.

L'histoire est aussi perçue comme un récit ${ }^{22}$. Antoine Compagnon ${ }^{23}$ voit le futur de l'histoire littéraire comme « une juxtaposition, une collation de textes fragmentaires liés à des chronologies différentielles... ». Telle en témoigne une série de billets de François Bon intitulés «Archéologie d'une lecture décisive » qui permet à l'écrivain, dans son actualité, de revisiter les notes écrites lors de sa lecture des œuvres qui ont compté pour lui ${ }^{24}$.

\section{Conclusion}

Il semble bien, en fonction de ces éléments d'analyse et de réflexion, que la critique littéraire exercée dans le cadre des blogs d'écrivains se soit effectivement reconfigurée sur Internet.

Les frontières entre les différentes catégories traditionnelles de critiques y apparaissent de plus en plus ténues : sur le blog du même écrivain alternent la critique journalistique, la critique d'auteur et la critique érudite.

La reconfiguration de la critique littéraire d'un point de vue communicationnel se vérifie dans l'interactivité créée avec les commentateurs qui laisse aux lecteurs la possibilité de participer, mais surtout dans les billets d'écrivains qui offrent à la fois des pratiques d'intertextualité et de « méta-

consensus, actes du colloque de Strasbourg du 12 au 17 mai 2003, Paris, PUF, 2005.

21. Rosa Galli Pellegrini, «Pour une histoire littéraire du contemporain : les entretiens d'auteurs ", L'Histoire littéraire à l'aube du vingtième siècle : controverses et consensus, actes du colloque de Strasbourg du 12 au 17 mai 2003, Paris, PUF, 2005.

22. Cf. Michel Foucault, «Faire l'histoire», Dits et Écrits, t. I, Paris, Gallimard, coll. Quarto, 2001; Paul Veyne, Comment on écrit l'histoire, Seuil, 1971; Jacques Rancière, Les Mots de l'histoire, Seuil, 1992; Paul Ricœur, Temps et Récit, Seuil, 1983.

23. Antoine Compagnon, « Du butinage numérique à l'écriture hypertextuelle », Le Monde, 5 mars 2009.

24. http://www.tierslivre.net. Des lectures d'Alain Robbe-Grillet, Claude Simon et Nathalie Sarraute. 
médiation », des savoirs littéraires offerts dans des langages véhiculés par des médias différents.

La critique littéraire exercée par ces écrivains relève de plusieurs couches génériques qu'il faut prendre en compte pour en définir les processus en cours.

Ces blogs se conjuguent sur deux temps, le passé et le présent. L'ensemble des textes assemblés sur ces dispositifs sont à la fois des archives et de l'actualité et concourent à une «redocumentarisation» du littéraire sur Internet. L'histoire littéraire devrait prendre en compte ces nouveaux phénomènes de reconfigurations de la critique, soit comme de nouvelles données, soit comme de nouvelles activités et pratiques littéraires en train de s'écrire.

Brigitte Chapelain Université Paris $13-L C P$

\section{Corpus des blogs d'écrivains}

Alcovère, Raymond, http://raymondalcovere.hautetfort.com/litterature/. Alpozzo, Marc, Marc Alpozzo (Ouvroir de réflexions potentielles), http:// marcalpozzo.blogspirit.com/.

Asensio, Juan, STALKER - Dissection du cadavre de la littérature, http:// stalker.hautetfort.com/.

Assouline, Pierre, La république des livres, http://passouline.blog.lemonde.fr/. Autié, Dominique, http://blog-dominique.autie.intexte.net/blogs/index.php/ all?cat $=16$ [blog fermé en mai 2008].

Barrière, Loïc, loicbarriere, http://loicbarriere.canalblog.com/.

Berthomet, Sébastien, http://berthomet-le-blog.blog.20minutes.fr/ [blog fermé en octobre 2009].

Bitton, Jean-Luc, Jacques Rigaut, http://www.rigaut.blogspot.com/.

Bleys, Olivier, Volubilis, http://bibliobleys.canalblog.com/.

Bon, François, Le tiers livre, http://www.tierslivre.net/spip/.

Castillo, Michel del, Le blog de Michel del Castillo, http://www.micheldelcastillo.com/index.php?option $=$ com_content $\&$ view $=$ category\&layout $=$ blo g\&id $=34 \&$ Itemid $=50$.

Cousin, Olivier, http://oliviercousin.over-blog.com/.

Fuentès, Roland, Brouillon littéraire et artistique, http://rolandfuentes.hautetfort.com/. 
Houdaer, Frédérick, Branloire pérenne, http://houdaer.hautetfort.com/ about.html.

Kuffer, Jean-Louis, Carnets de JLK, http://carnetsdejlk.hautetfort.com/.

Mabanckou, Alain, Le Crédit a Voyagé, http://www.lecreditavoyage.com/. Nohant, Gaëlle, Le café littéraire de Gaëlle, http://cafedegaelle.blogspot.com/. Parry, Patricia, Le blog de Patricia Parry, http://www.patriciaparry.com/. Rosnay, Tatiana de, Fig Tree : le Blog de Tatiana, http://yansor.blogs.psychologies.com/.

Sollers, Philippe, http://sollers.unblog.fr/.

Vebret, Joseph, http://www.vebret.com/.

Villemain, Marc, http://villemain.canalblog.com/. 